
e-ISSN 2594-0279 https://doi.org/10.33679/rmi.v1i1.1982

\title{
Pensando la inmigración desde las instituciones de la ciudad
}

Thinking Immigration from the City's Institutions

Kayamba Tshitshi Ndouba ${ }^{1}$

\section{RESUMEN}

Este artículo realiza una revisión bibliográfica de las formulaciones teóricas más destacadas que hacen dialogar los procesos migratorios con las dinámicas urbanas. De ella se desprenden dos registros de literatura académica. El primero, de corte sociológico, problematiza no solo las modalidades de la inserción progresiva de los inmigrantes en el espacio urbano, sino también los desafíos resultantes de las formas de ocupación y de transformación de los espacios sociales, económicos, culturales y simbólicos de la ciudad. El segundo, de corte politológico, profundiza en las nuevas perspectivas analíticas que recomponen y reformulan la problemática de gestión urbana de la inmigración, indagando en los distintos escenarios y factores que influyen y moldean la gobernabilidad de la inmigración y de la diversidad cultural en las ciudades. Los dos registros resultan de máxima utilidad y de valor heurístico innegable con el fin de construir una teoría moderna de las migraciones en el contexto urbano.

Palabras clave: 1. migración, 2. integración, 3. ciudad, 4. Europa, 5. América.

\section{ABSTRACT}

This paper conducts a bibliographic review of the main theoretical formulations that make migration processes dialogue with urban dynamics. From this, two records of academic literature arise. The first one, from a sociological approach, problematizes not only the modalities of gradual integration of immigrants into urban space but also the challenges resulting from the forms of occupation and transformation of the social, economic, cultural, and symbolic spaces of the city. The second one has a political science approach that explores the new analytical perspectives that reconstruct and reformulates the problems of the urban management of immigration, examining the different scenarios and factors that influence and shape the governance of immigration and cultural diversity in cities. These two approaches are significantly useful and have undeniable heuristic value to construct a modern immigration theory in the urban context.

Keywords: 1. migration, 2. integration, 3. city, 4. Europe, 5. America.

Fecha de recepción: 6 de diciembre de 2018

Fecha de aceptación: 11 de noviembre de 2019

Publicación en web: 30 de octubre de 2020

\footnotetext{
${ }^{1}$ Universidad Internacional de la Rioja-UNIR, España, kayamba.tshitshi@unir.net https://orcid.org/0000-0003-2582-7373
}

Migraciones Internacionales es una revista digital anual editada por El Colegio de la Frontera Norte. https://migracionesinternacionales.colef.mx 
$2 \begin{aligned} & \text { Pensando la inmigración desde las instituciones de la ciudad } \\ & \text { Tshitshi Ndouba, K. }\end{aligned}$

\section{INTRODUCCIÓN}

Los inmigrantes se incorporan a las instituciones democráticas y a la vida ciudadana de los países receptores desde su posición en el barrio, en el municipio y en la ciudad, lugares donde desarrollan cada una de las facetas de su vida. Las ciudades en particular son polos de atracción para las migraciones internacionales por el potencial económico, de competitividad y de innovación que ofrecen. Correlativamente, los crecientes y vertiginosos flujos de las migraciones internacionales hacia las ciudades favorecen la expansión urbana al mismo tiempo que plantean retos importantes de gestión de la diversidad cultural, de integración socioeconómica y de restructuración urbana (International Organization for Migration, 2015; Hatziprokopiou, Frangopoulos y Montagna, 2016).

Ahora bien, a pesar de su importancia como centros aglutinadores de las migraciones y como espacios de interacción y de conjunción inmediata de los intereses de las instituciones, de los ciudadanos autóctonos y de los nuevos vecinos, las ciudades y los municipios han ocupado una posición marginal en la exploración y en la elaboración de las teorías migratorias. La relación entre ciudad e inmigración, siendo una realidad tan antigua como la existencia misma de las ciudades (Portes, 2001), no acaba de situarse en un armazón analítico o cognitivo destinado a comprender, explicar y prever los procesos migratorios con una mirada integradora. Ello se debe a que durante mucho tiempo en todas las disciplinas sociales, la aproximación a la problemática migratoria se ha desarrollado desde los postulados del nacionalismo metodológico y desde las orientaciones de los estudios étnicos (Amelina, Nergiz, Faist y Schiller, 2012; Schiller, 2008). Desde esta perspectiva, la correlación normativa entre Estado e inmigración presupone que el Estado nación es la piedra angular del análisis social, puesto que es el modo de organización político moderno por excelencia, siendo intercambiables los conceptos de política y de Estado, siguiendo a los postulados weberianos (Dumitru, 2014).

La concentración espacial de la población inmigrante en las ciudades (y dentro de zonas específicas en las ciudades) intensifica los efectos sobre el entorno local, de modo que el impacto puede ser significativo, incluso en ciudades con una densidad de población inmigrante relativamente pequeña, afectando a un amplio abanico de ámbitos políticos locales como el mercado laboral local, la vivienda, la educación y otros servicios municipales (Alexander, 2003). Ello quiere decir que las dinámicas sociales, económicas y culturales derivadas del asentamiento de los inmigrantes en la ciudad generan una redefinición de las relaciones entre actores sociales, y conllevan reajustes en las políticas y servicios de las administraciones públicas locales, moldeando inevitablemente la gobernabilidad asimétrica de la inmigración en el ámbito local.

Partiendo de estas dos premisas, este artículo realiza una revisión bibliográfica de las formulaciones teóricas más destacadas que hacen dialogar los procesos migratorios con las dinámicas urbanas en dos registros. El primer registro, de corte sociológico, sintetiza la literatura académica más relevante que da cuenta de las complejas articulaciones entre la 
tríada urbanización, migración y desarrollo, problematizando las modalidades y los desafíos resultantes de la inserción progresiva de los inmigrantes en el espacio urbano. El segundo registro, de corte politológico, da cuenta de las nuevas perspectivas de análisis que recomponen y reformulan la problemática de gestión urbana de la inmigración, indagando en los distintos escenarios y factores que influyen y moldean la gobernabilidad de la inmigración y de la diversidad cultural en las ciudades receptoras de las migraciones internacionales.

\section{LA CIUDAD EN LAS TEORÍAS MIGRATORIAS}

La producción científica sobre las migraciones revela no solo la complejidad del objeto de estudio, sino también la inexistencia de unas teorías omnicomprensivas que abarquen todos los aspectos del fenómeno migratorio. Lo cierto es que existe una amalgama de explicaciones parciales que apuntan una constelación de teorías de grado intermedio, aun no imbricadas en una o varias teorías generales (Blanco Fernández de Valderrama, 1995). Desde los inicios de las investigaciones de la Escuela de sociología urbana de Chicago, la ciudad resultó ser un laboratorio de observación clínica de la vida social. Es por ello que, progresivamente, las ciencias sociales han ido construyendo hipótesis y herramientas conceptuales y metodológicas para explicar, interpretar y prever las interconexiones resultantes entre la ciudad y las oleadas de inmigrantes que recibe, así como las transformaciones y los cambios que se producen en la ecología de la ciudad.

A pesar de la multiplicidad de aproximaciones sobre la incorporación y la integración de los inmigrantes en la ciudad que se elaboran desde las diferentes disciplinas de las ciencias sociales, el entramado teórico sobre las interacciones entre la ciudad y sus inmigrantes adopta principalmente tres enfoques. El primer enfoque, de corte estructuralista, analiza la situación del inmigrante en la estructura social y en las demarcaciones espaciales de la ciudad, por comparación con los nativos, haciendo especial hincapié en procesos de integración/marginalidad residencial, económica, educativa y todo aquello relacionado con la estratificación y movilidad social. El segundo enfoque es de índole cultural y étnica, priorizando la situación cultural de la inmigración, desde la perspectiva de su situación minoritaria y marginal dentro de una comunidad dominante (mainstream society). El tercer enfoque es transnacional, puesto que ubica la inmigración y la ciudad dentro de los ámbitos globales del poder financiero, político y cultural y las trayectorias de salida y asentamiento de los inmigrantes, los cuales trascienden las fronteras estatales y crean "campos transnacionales".

\section{De la Escuela sociológica de Chicago al transnacionalismo}

En la tradición de la sociología americana y de la sociología de las relaciones interétnicas, la correlación entre ciudad e inmigración ha sido teorizada desde los prismas de producción y efectos de las etnicidades (Glazer y Moynihan, 1963), haciendo prevalecer el análisis de 
4 \begin{tabular}{l|l} 
Pensando la inmigración desde las instituciones de la ciudad \\
Tshitshi Ndouba, K.
\end{tabular}

las consecuencias y no de las causas de las migraciones en las ciudades. En efecto, los sociólogos de la Escuela de Chicago estructuraron la ciudad inmigrante como objeto de estudios sociológicos en torno a los postulados de la teoría general del cambio social, argumentando que la gran ciudad, por sus características objetivas y por la división de trabajo que impera en ella, define una nueva forma de existencia y de organización social y comunitaria, basada en la conciencia étnica (Park y Burgess, 1921). Desde sus inicios, la Escuela de Chicago se interesó por los aspectos de conformación de la cultura de la ciudad desde los supuestos del individualismo propio de una sociedad liberal.

La tradición sociológica de Chicago que aborda la relación migración-ciudad desde los paradigmas de asimilación y etnicidad ha sido cuestionada por las primeras teorías del transnacionalismo que emergen a partir de 1992, evidenciando nuevos perfiles de inmigrantes con fuertes vínculos con sus países de origen e inmersos en redes, actividades, estilos de vida e ideologías que sobrepasan las fronteras nacionales. En aquel momento, los trabajos pioneros de Schiller, Basch y Blanc (1992) ya habían puesto el énfasis sobre la intensidad, la frecuencia y la importancia cada vez mayor que adquieren las relaciones humanas de tipo material y simbólico que conectan las sociedades más allá de las fronteras nacionales. Sin embargo, de forma específica, en el transnacionalismo desarrollado por Schiller y Çăglar (2009) la dimensión de lo local (la ciudad) dentro de la migración transnacional adquiere una posición central de unidad de análisis. De esta forma, las dos autoras tratan de construir una teoría de la localidad para poder entender el fenómeno de la migración transnacional enfocando la localidad no como una cuestión de inmovilidad, sino como una cuestión de posicionamiento relativo dentro de ámbitos de poderes urbanos, estatales, regionales y globales. Sus teorizaciones recalcan la importancia que tienen las localidades particulares en la conformación de los procesos migratorios. Por ese motivo, proponen explorar la relación entre inmigración y localidad a partir del análisis del tipo de relaciones que surgen entre los residentes de una localidad y las instituciones de ámbito local, regional, nacional y global, observando especialmente cómo los migrantes contribuyen tanto a los procesos de desarrollo de una localidad como a su reconfiguración, reestructuración y re-escalamiento (Moraes Mena, 2004).

Así, el cuerpo teórico que elaboran Schiller y Çağlar (2011) proporciona herramientas valiosas que contribuyen a delinear las relaciones entre el tamaño, la importancia o la organización política de ciudades concretas receptoras de inmigrantes y las pautas de incorporación de las personas inmigrantes. En sus trabajos posteriores, sobre todo en la obra Locating Migration: Rescaling Cities and Migrants (Schiller y Çă̆lar 2011), abogan por considerar un enfoque combinado, que yuxtaponga estudios urbanos y estudios migratorios de cara a aprehender mejor y explicar el papel de los inmigrantes como artesanos y agentes del dinamismo de las ciudades en la era de la globalización. De tal forma, las dos autoras proponen una nueva visión comparativa que tenga como punto de partida las prácticas transnacionales de los inmigrantes y sus modos de incorporación a las ciudades. 
En la misma línea crítica de las teorizaciones de la Escuela de la sociología urbana de Chicago acerca del tándem ciudad-inmigración, cabe mencionar las valiosas contribuciones de Portes (2001). En efecto, al establecer una simetría entre la historia urbana al principio y al final del segundo milenio a cerca de la preferencia histórica de los migrantes hacia lugares de destino urbanos, y la transformación del tejido social y cultural de la ciudad como producto de tales flujos migratorios, Portes propone enfoques de análisis que sitúan la ciudad como centro neurálgico de la movilidad internacional de personas en busca de libertad política y de oportunidades económicas, desde las cuales se organizan las comunidades transnacionales de migrantes que reflejan un nuevo orden global. Desde estos planteamientos, las tesis de Portes establecen un nexo (o contigüidad) entre el proceso de identificación étnica y la asimilación en las instituciones de los Estados receptores de las migraciones internacionales.

La perspectiva crítica de Portes ha sido retomada por otros autores que se han dedicado a analizar las implicaciones económicas de la identificación étnica. Desde luego, el fenómeno de las economías étnicas o inmigrantes interesa -tanto desde una perspectiva económica como académica- debido a las importantes consecuencias laborales, financieras y sociales que se producen en los lugares donde se originan (Arjona Garrido y Checa, 2006). A este respecto, destacan las contribuciones de Light (1972), Light y Gold (2000), Light, Sabagh, Bozorgmehr y Der-Martirosian (1994), que sistematizan las condiciones de aparición y de consolidación de las actividades empresariales de coreanos, taiwaneses, japoneses, cubanos y afroamericanos en EE. UU. en sus áreas de asentamientos. En sus elaboraciones, de corte étnico-cultural, Light (1972) pone el énfasis en las prácticas de movilización de los recursos étnicos materiales (financiación), informacionales (consejos, orientación), y en la experiencia (formación) como factores justificativos del empresariado inmigrante.

Posteriormente a la teoría de las economías étnicas, Portes y Jensen (1989) emiten y examinan varias hipótesis sobre el perfil de los enclaves étnicos, los factores determinantes del autoempleo entre las minorías étnicas, así como los efectos de la participación en una economía de enclave étnico sobre los empresarios y los trabajadores. Los datos empíricos explorados apuntan dos conclusiones importantes sobre los enclaves étnicos: primera, las estructuras de los enclaves étnicos se definen por la proximidad física de las empresas de inmigrantes y no como meras aglomeraciones residenciales; segunda, los enclaves étnicos evitan los costes de emplearse en el mercado de trabajo secundario, al tiempo que constituyen una vía de movilidad social ascendente para los inmigrantes. Las elaboraciones sobre los enclaves étnicos han tenido el mérito de cuestionar el postulado de la teoría asimilacionista de la Escuela de Chicago, según el cual el mayor contacto entre los grupos inmigrantes y nativos de un territorio, promovido por los procesos de industrialización, tendría como efecto la desaparición de la solidaridad de tipo adscriptivo o mecánica (de grupo étnico) que sería sustituida por otra de tipo funcional u orgánica.

Ahora bien, a pesar de la proliferación de estudios sobre la economía étnica, no hay unanimidad entre los autores en cuanto a la tipificación de factores explicativos de la 
$6 \begin{aligned} & \text { Pensando la inmigración desde las instituciones de la ciudad } \\ & \text { Tshitshi Ndouba, K. }\end{aligned}$

aparición y consolidación del empresariado inmigrante. Por ejemplo, Waldinger (1985) apunta tres grandes líneas explicativas: la perspectiva culturalista, la ecológica (de los autores de la Escuela de Chicago) y la interaccionista. Este autor se sitúa él mismo en la corriente interaccionista, poniendo el acento en la compleja combinación entre tres dimensiones: las características del grupo (capacidad del grupo de movilizar recursos informales, disponibilidad de solidaridad familiar y comunal, etcétera), las estructuras de oportunidades (las condiciones de mercado, tipos de políticas gubernamentales, condiciones de concesión de licencias, etcétera) y las estrategias étnicas (modos de movilización de los recursos étnicos en el marco de unas estructuras de oportunidades concretas).

\section{Inmigración, ciudad y globalización}

Desde la última década del siglo XX, la literatura académica sobre la relación inmigraciónciudad está reorientando su foco de atención hacia la posición de las ciudades en el sistema migratorio global y sus consecuencias en la configuración del nuevo orden global del poder político, económico y cultural. Las contribuciones clásicas de Sassen (1991) han tenido el mérito de iluminar los procesos y las consecuencias de conversión de algunas ciudades en ciudades globales, auténticos centros de reorganización espacial de la economía mundial, caracterizadas por sus enormes capacidades de coordinación y de administración de las funciones de control y gestión de la economía capitalista global. Como tales ciudades, Sassen reconoce a Nueva York, Londres y Tokio. Estas ciudades se definen por una doble identidad: son a la vez unidades políticas subordinadas bajo particulares Estados nación y centros de mando y organización de una compleja economía mundial de la que depende considerablemente el futuro de esos mismos Estados nacionales (Portes, 2001). Otros autores han seguido los pasos de Sassen, tratando de profundizar en las "ciudades globales de segundo rango", que son las que asumen funciones de orden y control de la economía global capitalista a escala regional, siendo la ciudad de Miami, Florida, un caso paradigmático (Sassen y Portes, 1993).

Las investigaciones recientes de Schiller y Çağlar (2011) critican las perspectivas esencialmente orientadas a la exploración de los procesos de recomposición global del poder económico, político y cultural en los muros de "las ciudades globales" (global city), de "las ciudades globales de segundo rango" (midrange city) o de las "ciudades pasarelas" (gateway city). Por ello, las dos autoras desarrollan un enfoque centrado en el análisis de la relación entre, por un lado, los proyectos de regeneración urbana experimentados en algunas ciudades con diferentes grados de poder político, económico y cultural y, por otro lado, la sociabilidad cotidiana y las prácticas de ciudadanía social de todos los residentes de la ciudad. Los autores toman como caso de estudio tres ciudades con diferentes grados de similitud y de disimilitud en cuanto al poder político, económico y cultural: Mánchester, Nuevo Hampshire, EE. UU., Halle/Saale, Saxony-Anhalt, Alemania, y Mardin, Turquía. Este enfoque explora las potenciales interdependencias entre los procesos de desplazamiento, de reestructuración 
urbana y de la incorporación de los inmigrantes en esas ciudades. En este último aspecto, el enfoque parte del supuesto de que las relaciones entre estas ciudades y sus migrantes se habían convertido en una parte importante de los proyectos de regeneración urbana.

El enfoque metodológico utilizado por las dos autoras es el análisis multiescalar (multiscalar analysis). Descarta la dicotomía de niveles analíticos en los que el nivel macro del sistema mundial, o globalización, está por encima y más allá del nivel micro de la cotidianeidad. Rechaza también el escalamiento, en una jerarquía fija, de unidades territoriales delimitadas que van del barrio al Estado, pasando por ciudades y provincias. Partiendo de estas críticas, la metodología propuesta rastrea los procesos sociales y las diferentes esferas socioespaciales y sus interconexiones múltiples, tal y como se dinamizan en estas ciudades.

\section{Ciudades duales y lugares migratorios}

En otro registro, resaltan las investigaciones que se desarrollan en Europa desde la década de los ochenta del siglo XX, que enfatizan acerca de las dos caras de la inmigración en la ciudad: el crecimiento económico y la innovación frente a la exclusión social, la segregación y el conflicto. Este aspecto ambivalente de la inmigración en la ciudad justifica el apelativo de "ciudad dual", que Cachón (2010) utiliza profusamente para referirse a la integración espacial de la inmigración en las ciudades españolas. Este autor infiere que las ciudades españolas se están configurando en torno a dos factores determinantes que se interrelacionan entre sí: una realidad multicultural, caracterizada por la presencia en los barrios de personas que representan valores culturales diferenciados y entre las que no hay interrelación y comunicación, junto a otra realidad más física que presenta estructuras socioespaciales concretas, muy condicionadas por los procesos de inclusión social, el crecimiento y la generación de renta. La ciudad dual, marcada por el multiculturalismo y la exclusión, evidencia el surgimiento de barrios segregados.

Ahora bien, puesto que las migraciones son movimientos globales que nacen y aterrizan en algún lugar muy concreto, y que allí donde aterrizan ya hay sociedades en marcha, a menudo muy diferentes y complejas (Serra, 2007), los estudios migratorios empezaron a proponer algunas herramientas metodológicas y modelos teóricos para el análisis de la integración social de los inmigrantes en las ciudades. En España, el modelo de Integración Social de Inmigrantes (ISI) propuesto por Izquierdo Escribano y Noya (1999) tiene un valor heurístico innegable. Este modelo desarrolla un enfoque meso, que toma en cuenta el entorno material y social en el que se produce la integración social de los inmigrantes. La propuesta incorpora como variables centrales "los contextos concretos de la inmigración" (modalidades de zonas de residencia, tipos de organizaciones o ámbitos en el que se trabaja), sobre los que se aplica una metodología cuantitativa de análisis multinivel, así como "la densidad de las redes sociales" (capital social y relacional), que se interpreta desde la metodología de análisis de redes. 
La esfera de análisis substantivo donde puede aplicarse empíricamente el enfoque metodológico del modelo de integración social de inmigrantes (ISI) propuesto por Izquierdo Escribano y Noya son los lugares migratorios, que los dos autores definen como "contextos particulares, espacios concretos en los que se han sedimentado olas migratorias de distinta índole: interiores, de retorno e internacionales", y por ende, son " espacios caracterizados por una alta densidad y variedad de flujos migratorios" Izquierdo Escribano y Noya (1999, p. 34). El análisis de las estructuras sociales y de los vínculos sociales y culturales generados en los "lugares migratorios" puede dar pistas interesantes en aras de interpretar los procesos urbanos de acogida, movilidad y marginación que se producen en algunas ciudades. Basándose en las investigaciones realizadas en otros contextos particulares en los que se dan una diversidad cultural de gran envergadura, como por ejemplo el barrio de Bijlmermeer, en Ámsterdam, Izquierdo Escribano y Noya (1999) establecen como hipótesis de partida que la variable contextual de los lugares migratorios (acumulación y variedad cualitativa de situaciones migratorias y, por ende, de relaciones sociales en una zona) influye en el clima social, elevando sensiblemente el umbral de tolerancia y fluidificando la integración cultural. Los estudios anteriores a la propuesta de estos dos autores localizan los "lugares migratorios" españoles en Galicia, Andalucía y Cataluña.

\section{Ciudades del sur de Europa: diversidad, economía étnica y segregación espacial}

El cambio del estatus migratorio de los Estados del sur de Europa, al convertirse en espacios geográficos receptores de la inmigración a partir de la última década del siglo pasado, ha generado una nueva revisión de los postulados teóricos y de las hipótesis sobre las pautas de asentamiento residencial de los inmigrantes y de los modos de segregación étnica imperantes en las ciudades americanas y de Europa del norte. Por ello, desde la primera década del siglo XXI está aflorando otra agenda de investigación de corte demográfico y de geografía urbana. Las distintas líneas de esta agenda despliegan conceptos analíticos, herramientas metodológicas y enfoques de estudio, poniendo particular énfasis en los procesos de marginalización, vulnerabilidad y exclusión social de los inmigrantes en la ciudad a partir de las dimensiones de integración espacial (dispersión, concentración o segregación en cuanto a la residencia) y de la lucha contra las discriminaciones (Echazarro de Gregorio, 2009; Pfirsch y Semi, 2016).

En particular, las investigaciones de Arbaci (2008) y de Malheiros (2002) exploran la coincidencia entre los problemas y las causas de la segregación residencial y los procesos diferenciación étnica y social en las ciudades mediterráneas. Las investigaciones realizadas infieren que la desconcentración geográfica, como pauta de asentamiento de los inmigrantes en las ciudades del sur de Europa, es un síntoma negativo de inserción étnica. La dispersión geográfica de los asentamientos inmigrantes a lo largo y ancho de la ciudad marca la marginación residencial. No se trata de un efecto coyuntural, sino que forma parte de mecanismos estructurales derivados de un proceso amplio de segmentación del mercado 
laboral y sobre todo, de división social del espacio y el predominio de la vivienda en propiedad, difícilmente accesible para los inmigrantes laborales.

Otros estudios sobre la integración de la inmigración en las ciudades del sur de Europa enfatizan sobre el papel del empresariado étnico en la reconfiguración urbana, situando la economía étnica en la intersección entre las tendencias de asentamiento de inmigrantes y los procesos de cambios que experimentan las ciudades. Este enfoque, liderado por las investigaciones de Hatziprokopiou y sus colegas (Hatziprokopiou et al., 2016), toma como caso de estudios empíricos las ciudades de Atenas, Milán y Viena. Estos autores critican las perspectivas clásicas que enfatizan solo en los aspectos socioeconómicos y étnicos del empresariado étnico, poniendo de manifiesto la idoneidad de enmarcar el empresariado étnico en las dinámicas de cambio social y de transformación urbana.

\section{Ciudad, asilo y hospitalidad}

Con la crisis de refugiados, agudizada en la Unión Europea entre los años 2014 y 2016, ha resurgido no solo el debate político sobre los límites de la arquitectura del Sistema Europeo Común de Asilo (SECA), sino también el interés académico por interrogar el papel de las ciudades como marco de desarrollo de las políticas de acogida y de socialización de refugiados.

Los procesos de acogida e integración de los refugiados en las ciudades europeas han sido ampliamente problematizados a través de la agenda de investigación del proyecto "Refugees in European localities: Reception, Perceptions and Policies (RELOCAL)", desarrollado por la red International Migration, Integration and Social Cohesion (Imiscoe). Las investigaciones de este proyecto profundizan en los debates y controversias académicas en torno a tres ejes resultantes del binomio ciudad-refugiados.

Primero, se plantea la cuestión de identificar los enfoques teóricos más apropiados para enmarcar y analizar los procesos de acogida e integración de los refugiados, evitando las categorías políticamente definidas en el diseño y planteamiento metodológico de la investigación. Segundo, partiendo del supuesto de que las ciudades son los escenarios de contacto e interacción inmediata entre la populación autóctona y los flujos de refugiados que aterrizan en los espacios urbanos, se plantea la necesidad de analizar empíricamente no solo las condiciones en las que surgen los conflictos locales sino también los modos de conceptualización y de resolución de dichos conflictos. Por último, interesa ahondar en el tipo de discurso público que desarrollan las autoridades locales en torno a los problemas que plantean la acogida e integración de los refugiados en la ciudad, por una parte, y por otra, las respuestas ciudadanas de los lugareños y la capacidad auto- organizativa de los propios solicitantes de asilo y refugiados en la ciudad. En estos aspectos, destaca la obra editada por Doomernik y Glorius (2016): "Refugee Migration and Local Demarcations: New Insight into European Localities" que proporciona interesantes evidencias empíricas de las 
problemáticas locales en torno a la acogida e integración de los refugiados en las diferentes ciudades de cinco Estados: Alemania, Reino Unido, Noruega, Italia, Turquía y Bulgaria.

Asimismo, conviene resaltar que desde la segunda década del siglo XXI emerge otra categoría de fecunda literatura académica sobre las ciudades y refugiados que se desarrolla en dos registros. El primero profundiza en los dilemas y los debates sobre "los espacios de refugiados" (refugee spaces). Destaca el interesante artículo de Sanyal (2012) titulado Refugees and the City: An Urban Discussion, que sintetiza acertadamente los problemas inherentes a la política de "urbanización de los campos de refugiados" y las implicaciones de la gestión de la atención humanitaria a los refugiados asentados dispersamente en los barrios marginales y las periferias de grandes ciudades. El segundo gira en torno a la tríada: ciudad, asilo y hospitalidad, poniendo de relieve la dimensión solidaria de una constelación de "villes-refuges", "ciudad de asilo", "sanctuary-city" que proliferan en algunos Estados de Europa y de América del norte. Los trabajos de Filomeno (2018), Furri (2017), Delgado (2018), Aparna y Schapendonk (2018) y finalmente Darling (2017), retratan las tensiones políticas resultantes de la resistencia de las ciudades a las prescripciones del marco de elegibilidad y de concesión del derecho de asilo, las trasformaciones de las ciudades refugios/suntuarias y las negociaciones entre los diferentes actores urbanos en la producción de los imaginarios y el relato de la hospitalidad.

\section{LAS RESPUESTAS LOCALES AL HECHO MIGRATORIO DESDE LOS ENFOQUES POLITOLÓGICOS}

El desplazamiento del interés por el marco nacional hacia la importancia del territorio y del régimen local como unidad de análisis de los fenómenos migratorios, encauza los debates académicos sobre los enfoques más idóneos para abordar los problemas y las soluciones locales al hecho migratorio. Los extremos de este debate van conformando un cuerpo teórico de las políticas locales de inmigración, que Filomeno (2016) sintetiza y desglosa en dos enfoques opuestos: uno localista y otro relacional.

De acuerdo con el primero, los problemas locales asociados con la inmigración impulsan políticas concretas que son moldeadas por las condiciones particulares y tienen resultados precisos. Las condiciones locales enfatizadas son: la identidad y composición étnica de la comunidad, la competencia entre inmigrantes y nativos por los recursos, la política electoral y el partidismo, y la burocracia y la movilización de las organizaciones de apoyo a los inmigrantes. Los resultados de políticas locales son la organización cívica de las comunidades locales de inmigrantes, el nivel de actividad económica y la seguridad pública municipal.

En contraste con el enfoque localista, los partidarios del enfoque relacional postulan que la política local de inmigración se gesta desde arriba hacia abajo, abarcando procesos que entrecruzan y enlazan múltiples localidades. Siguiendo esta orientación, las administraciones locales no solo siguen las directrices nacionales, incluso pueden influir en 
la gobernanza de la inmigración a nivel nacional. Las relaciones horizontales entre los gobiernos locales, incluyendo la competencia y la cooperación, también se consideran importantes para la formulación y la aplicación de políticas locales para los inmigrantes. Estas interacciones verticales y horizontales son cruciales no solo para explicar la política de inmigración local, sino también para entender por qué las políticas convergen o divergen en la gobernanza multinivel de la inmigración.

La exposición de los dos enfoques - localista y relacional- propuestos por Filomeno (2016) demuestra la mayor relevancia que tienen las líneas de investigación, de corte politológico e interesadas en analizar los "arreglos institucionales", en la formulación y el desarrollo de políticas migratorias entre una multiplicidad de actores políticos. La articulación y la coordinación de todos los actores participantes en este gobierno multinivel de la inmigración es una dificultad añadida a la complejidad y a la transversalidad de las políticas migratorias. Lo paradójico es que, pese a que las políticas de inmigración se determinan, diseñan y financian a nivel nacional y europeo, la intervención local y urbana es esencial para lograr la integración de los inmigrantes internacionales, ya que el impacto de dichas políticas, tanto en los inmigrantes como en la sociedad, se experimenta a nivel local, donde interaccionan otras áreas de actuación como las del mercado de trabajo y del desarrollo económico.

Por ello, en las últimas décadas, la importancia del ámbito local en los procesos migratorios inspira a muchos estudios y se desarrollan distintas líneas de investigación interesadas en analizar las dinámicas y las consecuencias de las interconexiones verticales propias de un gobierno multinivel, con las autoridades de la UE, nacionales, regionales y locales (Dekker, Emilsson y Krieger, 2015; Jørgensen, 2012; Penninx y Garcés-Mascareñas, 2016; Scholten y Penninx, 2016). Una visión global de estos trabajos revela un esfuerzo por elaborar hipótesis y enunciados teóricos a partir de varios registros de prácticas y experiencias locales de la relación jurídica, administrativa y política entre el Estado y la ciudad. Algunos trabajos parten del análisis de las lógicas divergentes entre las políticas migratorias nacionales y locales (Jørgensen, 2012) o toman en cuenta el impacto de las legislaciones nacionales en los contextos locales concretos (Caponio, 2005; Emilsson, 2015). Esta línea de investigación incluye también los estudios que comprenden los efectos y la retroalimentación recíprocas entre los dos niveles de gobierno: el Estado y la ciudad (Scholten, 2016; Scholten y Penninx, 2016). Esta categoría encierra también algunos trabajos que ponen foco a otros factores propiamente locales, como la relación estructural entre el gobierno estatal y el gobierno local, que configura el marco y la esencia de las políticas locales de integración de los inmigrantes (Garbaye, 2000; Scholten, 2013).

Por último, la gobernanza de la inmigración y de la diversidad en las ciudades ha sido ampliamente desarrollada por varios autores reunidos en la obra editada por Caponio, Scholten y Zapata-Barrero (2019). Los capítulos expuestos ofrecen una visión interdisciplinaria de la problemática de la gestión urbana de la inmigración al mismo tiempo propone una agenda de investigación sobre la temática. Tiene el mérito de componer un 
$12 \begin{aligned} & \text { Pensando la inmigración desde las instituciones de la ciudad } \\ & \text { Tshitshi Ndouba, K. }\end{aligned}$

plano de los posibles factores y procesos que influyen y dan forma a la gobernabilidad de la migración y la diversidad en las ciudades, desde la comprensión histórica y sociológica de la gobernanza urbana.

\section{Inmigración, Ciudad y Estado: hacia el giro local (local turn) de las políticas migratorias}

El retroceso de la hegemonía del nacionalismo metodológico y del paradigma de etnicidad en los estudios migratorios coincide con la adopción progresiva de la perspectiva del "territorio" o de la "localidad" y de los actores locales, desplazando las fronteras sociales y geográficas (o físicas) de la investigación hacia demarcaciones locales y regionales. Con ello interesa también la posición de la "localidad" en los espacios de movilidad y de los intercambios materiales y simbólicos transnacionales. Este nuevo impulso académico hacia los procesos de territorializacion de la inmigración infiere la importancia del contexto local como el marco que acoge la dimensión real del asentamiento de los inmigrantes y, por ende, como marco institucional básico en el que se establecen las políticas migratorias (Fauser, 2008). Esta nueva orientación profundiza también en los cambios que se producen en la gobernanza local de la inmigración desde las dinámicas de reestructuración del "subsistema de actores" que "hacen y confeccionan" el territorio.

Asimismo, la perspectiva del "territorio" otorga un papel significativo a la posición y a la acción de la propia comunidad inmigrante en los circuitos institucionales y los procesos de toma de decisiones políticas, examinando las múltiples interrelaciones que establecen los actores, cómo se perciben mutuamente, se oponen, realizan alianzas, se imponen criterios y finalmente, organizan el territorio (Moine, 2006). El análisis de la acción "situada" de los actores locales tiene la ventaja de ofrecer una visión sistemática de las interacciones entre las diferentes estructuras y agentes involucrados en las respuestas locales a los retos que plantea la inmigración y la diversidad.

Ahora bien, conviene anticipar que cuando nos referimos al ámbito local, aludimos, desde luego, a la unidad básica de la administración local: el municipio. Pero además de él pueden existir niveles de segundo grado que agrupan a varios municipios, incluso entes inframunicipales (Baena del Alcázar, 2000). El giro local (local turn) implica reconocer que ciertos aspectos vinculados a la gestión migratoria, por su naturaleza, escapan al control del gobierno central, induciendo la idea de cooperación y de coordinación con otros actores: las autoridades locales y la sociedad civil. Ello significa que el desarrollo y las consecuencias de las políticas locales de inmigración no pueden entenderse de forma aislada, es decir, al margen de su contexto. Las relaciones intergubernamentales, la globalización económica y las instituciones internacionales son variables y condicionantes cruciales que limitan considerablemente la autonomía local en la gestión del hecho migratorio (Schoten, 2019).

Para ahondar en la posición institucional de las administraciones locales en las políticas migratorias, algunas investigaciones han puesto el foco en el grado de autonomía local de cara a la integración de los inmigrantes y las dimensiones locales de dicha integración. A 
estas alturas, conviene precisar que si bien en el sistema anglosajón los municipios y otros entes locales son considerados "gobiernos locales", y en consecuencia tienen una autonomía según la peculiar acepción de este término en aquel sistema (self goverment), en el sistema continental europeo los entes locales no son gobierno sino administración, y como tal forman parte de la estructura general de la administración de cada Estado (Baena del Alcázar, 2000).

En todo caso, a pesar del giro local que experimentan las políticas migratorias, resulta muy difícil conceptualizar un campo político local, tal y como ha sido teorizado por Bourdieu (2012), circunscrito a las migraciones, puesto que en la gestión de esta materia imperan más los principios de coordinación y cooperación entre todos los actores. Aún más, por lo general, el ámbito local (ciudades y municipios) no dispone de competencias privativas en materia de integración de los inmigrantes. Desde lo local se desarrollan transversalmente ciertas competencias para una mejor integración en relación a la planificación urbana, la cohesión social (reducir las desigualdades sociales y urbanas, así como los fenómenos de exclusión), la educación o la sanidad desde los prismas de competencias en relación con los residentes empadronados en la ciudad, atendiendo siempre a los principios de normalización y de igualdad de trato.

El análisis pormenorizado del ámbito competencial de las ciudades en lo relativo a la integración de los inmigrantes ha desembocado en dos tipos de tratamientos en la literatura politológica europea: por una parte, las formulaciones teóricas que se focalizan en las relaciones verticales "multinivel" entre los distintos actores políticos y sus implicaciones en el desarrollo de las políticas de integración, y por otra parte, las formulaciones que examinan y teorizan las respuestas locales a las migraciones internacionales desde la perspectiva de la administración pública. Estos dos enfoques se imbrican en muchos aspectos y son complementarios.

\section{Ciudad y Estado. Escenarios y dilemas de la gobernanza "multinivel"} de la inmigración

En el primer perfil del tratamiento académico del gobierno multinivel de la inmigración, la problemática central de la investigación se interesa en saber de buena tinta de qué forma y por qué las diferentes configuraciones de la relación entre los gobiernos centrales y locales afectan el modo de gobernanza de las políticas de integración de los inmigrantes. En esta línea de investigación, destacan los trabajos de Peter Scholten y sus colegas (Caponio et al., 2019; Scholten, 2016; Scholten y Penninx, 2016) y Henrik Emilsson (2015) profundizan en la temática desde un enfoque comparativo.

Scholten $(2011,2013,2016)$ aborda los dilemas y los distintos escenarios (alineamiento o asimetría) de una gobernanza multinivel de la inmigración comparando dos patrones divergentes de estructuras de gobernanza y de modelos políticos de integración de los inmigrantes en Reino Unido y en Holanda. Para el logro de sus propósitos, selecciona dos ciudades de cada país, conocidas por sus enfoques divergentes: Londres (los distritos de 
$14 \begin{aligned} & \text { Pensando la inmigración desde las instituciones de la ciudad } \\ & \text { Tshitshi Ndouba, K. }\end{aligned}$

Tower Hamlets y Enfield) y Glasgow por una parte, y por otra, Rotterdam y Ámsterdam. El estudio de los casos empíricos escogidos le lleva a concluir que no existen mecanismos de coordinación propios de un enfoque "top-down" que aseguren un marco de lineamiento simétrico que requiere formatear un modelo nacional de integración de los inmigrantes. Reino Unido enfatiza un marco de lineamiento y criterios comunes en torno a ciertas iniciativas específicas, dejando a los gobiernos locales ordenar sus propias definiciones y enfoques de integración a través de recursos jurídicos y políticos, optimizando en algunos casos los instrumentos de autogobierno y forzando una disposición asimétrica. En cambio, Holanda carece de un sistema vertical centralizado de gobierno multinivel. Ello conlleva a un escenario de marcos divergentes y asimétricos que, a veces, acarrea marcos políticos conflictivos y contradictorios.

Los trabajos de Emilsson (2015), comparando las dinámicas de la gobernanza multinivel de las políticas de integración en Suecia y Dinamarca, son muy representativos de la problemática de cooperación y coordinación del gobierno multinivel de la inmigración. Para este autor, aunque los gobiernos locales se han convertido en importantes actores de la política de integración, las ultimas tendencia de las políticas migratorias de Dinamarca y Suecia demuestran un giro nacional ("a national turn of local integration policy") puesto que los gobiernos centrales de ambos países han logrado aumentar su control e influencia a nivel local, lo que debilita la capacidad de los gobiernos locales de formular sus propias políticas de integración. Haciendo hincapié en el nuevo rumbo iniciado de políticas migratorias, Emilsson constata que, a pesar de sus enfoques diferenciados, los dos Estados han cambiado de paradigma político, incrementando su control y grado de autoridad sobre los gobiernos locales, recentralizando los instrumentos de gobernanza (blandos) y de control (gobierno).

En la misma línea que los dos autores, cabe recalcar las investigaciones cuyo argumento principal gira en torno al marco teórico adecuado para explicar estas divergencias en los distintos niveles del gobierno de la inmigración (Estado-ciudad). A este respecto, Jørgensen (2012), al comparar los enfoques de políticas migratorias aplicados en cuatro municipios daneses (Aalborg, Arhus, Copenhague y Thisted), sostiene que los conceptos de "lógicas institucionales" y "racionalidades políticas" pueden ser combinados con las nociones de estructuras de oportunidad política (Political Oportunity Structure-POS). Desde este marco teórico, el autor argumenta que las divergencias entre los niveles de gobierno central y del gobierno de la ciudad pueden encontrar una explicación no solamente como fruto de una transposición insatisfactoria de las políticas nacionales en el ámbito local, sino también puede considerarse como el resultado de una divergencia de los marcos políticos alternativos en situación de competición, de la racionalidad política y de lógicas institucionales. 


\section{Inmigración y autonomía local: la gobernanza asimétrica de la inmigración}

Por lo general, en el entramado de la red de actores, el Estado o gobierno central ostenta el papel de dirección política al enunciar los objetivos de las estrategias públicas de integración de los inmigrantes y al regular legislativa y administrativamente sobre los instrumentos básicos de financiación y de ejecución de las políticas de integración. La falta de una potestad de libre decisión, así como la falta de "reserva" de un ámbito de competencias sustantivas no susceptibles de limitación por el legislador nacional en lo referente a la acogida e integración de los perfiles migratorios, imposibilita el desarrollo de unos instrumentos perentorios que sirvan de plataforma para "unas políticas públicas locales" que puedan ser circunscritas como objeto específico de estudio.

Lo cierto es que los estamentos locales carecen de un cuerpo normativo autónomo acerca del tema migratorio y de una filosofía pública de integración que inspiren sus acciones, aunque puedan disponer de recursos económicos y de instituciones políticas y administrativas pensados para poner en marcha las actuaciones de la administración local. Por otra parte, las administraciones locales están en faltas de unos recursos materiales y financieros autónomos y suficientes para responder adecuadamente a los retos de integración en el ámbito local. A partir de estos supuestos, se desarrollan dos registros importantes de investigación que profundizan en la posición institucional de las administraciones locales en las políticas migratorias.

El primer registro se centra en el análisis del grado de autonomía política y administrativa de las administraciones locales para desarrollar una política de integración de los inmigrantes. Destacan los esfuerzos de algunos autores por establecer una tipología de enfoques locales de políticas de integración (Alexander, 2003; Pennix y Martiniello, 2006). En particular, Alexander (2003), inspirado por la triple clasificación de políticas migratorias elaborada por Castles (1995) -exclusión diferencial, asimilacionista y pluralista- postula cuatro tipos o fases de actitudes o posicionamiento de las autoridades municipales con respecto a los migrantes y su alteridad, las cuales se materializan en políticas específicas hacia la población migrante en el ámbito local. Las actitudes de las autoridades se categorizan en dos casillas: una modernista y otra postmodernista.

Desde el punto de vista modernista se asume que la presencia del inmigrante en el municipio es transitoria o temporal y se adoptan dos posturas diferenciadas, pero que se integran en una misma filosofía pública de marginalidad o segregación: en la primera postura, se adopta una actitud de no política. La segunda postura se refiere a la consideración de trabajador invitado, guest worker, y se caracteriza por una actitud de tolerancia (no entusiasta). Las dos posturas modernistas coinciden en ignorar la presencia del inmigrante y no tener en cuenta su alteridad. En cambio, desde la posición postmodernista se asume que la presencia del inmigrante es permanente. En este caso, la reacción política puede ser asimilacionista, pretendiendo reducir o eventualmente eliminar la alteridad (incluida la segregación) o pluralista, tolerando y posiblemente apoyando la alteridad, incluida una 
cierta tolerancia a la segregación. Observamos entonces que los cuatro tipos posibles de actitudes se traducen en posibles políticas municipales: transitorio (no-política), trabajador invitado, asimilacioncita y pluralista. Cada una de estas actitudes constituye una fase de las políticas locales, que siendo dinámicas pueden evolucionar. Alexander (2003) identifica, además, tres variables importantes que condicionan el desarrollo de las políticas locales de inmigración: las trayectorias de desarrollo de políticas municipales/urbanas, el predominio o la priorización de un ámbito político sobre otro (por ejemplo ciudades francesas se centran en la politique de la ville mientras que las ciudades británicas se centran sobre las race relations) y el tipo de relaciones ciudad-Estado.

Para completar el análisis de la posición institucional de las administraciones locales en los procesos de integración de los inmigrantes, es necesario apuntar también los trabajos empíricos sobre las políticas locales realizados en el marco del proyecto Knowledge for integration governance (KING) (Gilardoni, D’Odorico y Carrillo, 2015) y la obra de Rinus Penninx (2009), miembro del cuerpo asesor del proyecto KING, destacando tres ámbitos o dimensiones de integración de los inmigrantes en el ámbito local: 1) socio-económica, 2) cultural y cohesión social y 3 ) legal y política.

En la misma línea de investigación, destacan también las perspectivas institucionales desarrolladas por Penninx y Martiniello (2006). Los dos autores dan cuenta de los debates y controversias académicas en torno a dos tipos de instituciones especialmente relevantes en los procesos de integración de los inmigrantes en el ámbito local: las que poseen un carácter universal y aquellas que existen "por y para" los grupos inmigrantes. Las instituciones universales se dirigen a todos los ciudadanos de forma igualitaria: el sistema educativo, los arreglos institucionales en el mercado de trabajo, el sistema de salud pública o el mismo sistema político, por ejemplo. Los dos autores argumentan que las políticas de integración deberían tener en cuenta la totalidad de las dimensiones o los ámbitos que cubren, lo que significa que no solo deberían reflejar las inquietudes de la mayoría autóctona, sino también ser sensibles a las necesidades específicas de los grupos inmigrantes y adaptarse a las condiciones locales. Por otra parte, los dispositivos de atención especializada creados "por y para" los grupos inmigrantes, se enmarcan también en el contexto de la calidad democrática y de la gobernanza local, puesto que ponen énfasis en el papel de los actores (políticos y stakeholders) en el doble ámbito del proceso de toma de decisión (decisonmaking process) y de la implementación administrativa de las decisiones políticas.

El segundo registro de investigaciones enfocadas a las dinámicas entre inmigración y autonomía local problematiza especialmente las tensiones del gobierno multinivel de la inmigración, intentado teorizar las respuestas locales a las migraciones internacionales desde la perspectiva de la administración pública. En España, las contribuciones de Zapata-Barrero (2012) presentan un interés teórico de gran calado. En efecto, este autor analiza en profundidad los aspectos de la autonomía local de cara a los nuevos retos locales en el tema migratorio, argumentando que de acuerdo con el principio de proximidad y de 
subsidiariedad, las administraciones locales empiezan a activar desde 2011 sus recursos legales (ordenanzas), administrativos (gestión del empadronamiento) y políticos (acogida, gestión del pluralismo religioso y del espacio público, gestión de discursos y conflictos sociales) para conformar sus perspectivas más inmediatas. Todo ello sin tener en cuenta una visión global, ni la coherencia de políticas, ni los efectos que puede tener sobre la propia dinámica migratoria. Este autor categoriza un tripe papel que las administraciones locales pueden activar, con diferentes grados de importancia. El papel activo autónomo como gestor de las políticas relacionadas con diversidad y aspectos técnico-administrativos (empadronamiento), el papel de entidad gestora (que administra decisiones de otros Gobiernos superiores y colabora en políticas de otros niveles de Gobierno) y papel territorial (la ciudad territorializa sus políticas, decide políticas y establece "fronteras locales" que afectan directamente la movilidad de los inmigrantes).

\section{CONCLUSIONES}

Los crecientes y vertiginosos flujos variados de migraciones internacionales hacia las ciudades han llevado a la comunidad científica a cuestionar los conceptos, las categorías y las herramientas para pensar acerca de las relaciones entre la inmigración y la reconfiguración del tejido social, económico y cultural de las ciudades. Además del interés clásico por analizar las formas de inserción de los inmigrantes en el espacio urbano, las recientes contribuciones académicas sobre la relación inmigración-ciudad están reorientando su foco de atención hacia dos campos.

Por una parte, las políticas locales de integración de los inmigrantes y sus vínculos con las políticas estatales con el objeto de analizar los factores y dinámicas institucionales que intervienen en la concepción y la implementación de los mecanismos y "arreglos institucionales" para la integración de los inmigrantes impulsados por las ciudades receptoras de las migraciones. Por otra parte, la posición de las ciudades en el sistema migratorio global, intentando ensanchar el espacio de intersección y de convergencia entre los estudios migratorios y los estudios urbanos. Con ello, el conjunto de contribuciones encauza el desarrollo de una perspectiva comparativa de categorización de modelos locales de gestión del hecho migratorio en el ámbito urbano, que a su vez resulta de máxima utilidad en la construcción de una teoría moderna de migraciones en el contexto urbano, integrando problemáticas propias de la historia, la antropología, la sociología, la geografía y el urbanismo, como cuestiones centrales de la época contemporánea.

La revisión bibliográfica destaca dos aspectos muy relevantes de cara al andamiaje teórico de alcance intermedio sobre los problemas y las respuestas a la integración de la inmigración en la ciudad. Primero, la relevancia de la estructura social, económica y política del "territorio" donde aterrizan y confluyen las migraciones, lo cual convierte la ciudad en un campo fértil para reelaborar las teorías migratorias. Segundo, las diferentes aproximaciones a la problemática de la inmigración en la ciudad enfatizan en la necesidad de contextualizar 
cada caso concreto, puesto que los movimientos migratorios alinean las ciudades en lugares abiertos para procesos de alcance regional, nacional y transnacional. En consecuencia, la diversidad y la complejidad de los procesos de reconfiguración "territorial" urbana por los profundos influjos de la globalización de la economía, de la transformación del poder político del Estado y del cambio de paradigma de la movilidad humana crean cada vez dinámicas novedosas y cambiantes, a la vez que "situadas" y específicas.

Asimismo, el valor heurístico de la posición de las ciudades en la configuración del nuevo orden migratorio de la era de la globalización justifica la necesidad de seguir produciendo evidencias empíricas con relación al papel de la inmigración en la restructuración de las ciudades de diferentes tamaños, diferentes grados de diversidad cultural y de posiciones y escalamientos geopolíticos regionales diferentes. Y, sobre estas bases, extraer elementos de similitud, disimilitud y grados de variedad que proporcionen los fundamentos esenciales para analizar, interpretar y explicar no solo los procesos de acogida e integración de los inmigrantes en las ciudades, sino también para comprender los dilemas y escenarios de las respuestas a los retos migratorios impulsadas desde las ciudades y desde sus instituciones.

\section{REFERENCIAS}

Alexander, M. (2003). Local policies toward migrants as an expression of Host-Stranger relations: A proposed typology. Journal of Ethnic and Migration Studies, 29(3), 411-430. https://doi.org/10.1080/13691830305610

Amelina, A., Nergiz, D., Faist, T. y Schiller, N. G. (2012). Beyond Methodological Nationalism: Research Methodologies for Cross-Border Studies. Nueva York: Routledge.

Aparna, K. y Schapendonk, J. (2018). Shifting itineraries of asylum hospitality: Towards a process geographical approach of guest-host relations. Geoforum. https://doi.org/10.1016/j.geoforum.2018.03.024

Arbaci, S. (2008). Hacia la construcción de un discurso sobre inmigración en las ciudades del sur de Europa. La política urbanística y de vivienda como mecanismos estructurales de marginación étnica residencial. Architectute City and Environement, 8, 11-38.

Arjona Garrido, A. y Checa, J. C. (2006). Economía étnica. Teorías, conceptos y nuevos avances. Revista Internacional de Sociología, 64(45), 117-143.

Baena del Alcázar, M. (2000). Problemas del Gobierno local en España (con especial referencia a la organización administrativa de los entes locales). Cuadernos de Gobierno y Administración, 1, 11-22.

Blanco Fernández de Valderrama, C. (1995). La integración de los inmigrantes en las sociedades receptoras: Método de análisis y aplicación al País Vasco. Tesis doctoral. Facultad de Ciencias Políticas y Sociología. Bilbao: Universidad de Deusto.

Bourdieu, P. (2012). Intelectuales Cultura y poder. Madrid: Clave Intelectual. 
Cachón, L. (2010). Informe sobre la situación de la integración social de inmigrantes y refugiados en España 2009. Madrid: Ministerio de Trabajo e Inmigración.

Caponio, T. (2005). Policy Networks and Immigrants' Associations in Italy: The Cases of Milan, Bologna and Naples. Journal of Ethnic and Migration Studies, 31(5), 931-950. https://doi.org/10.1080/13691830500177891

Caponio, T., Scholten, P. y Zapata-Barrero, R. (2019). The Roulledge handbook of The govennance of Migration and diversity in cities. Londres/Nueva York: Routledge.

Castles, S. (1995). How nation-states respond to immigration and ethnic diversity. New Community, 21(3), 293-308.

Darling, J. (2017). Forced migration and the city: Irregularity, informality, and the politics of presence. Progress in Human Geography, 41(2), 178-198. https://doi.org/10.1177/0309132516629004

Dekker, R., Emilsson, H. y Krieger, B. (2015). A Local Dimension of Integration Policies? A Comparative Study of Berlin, Malmö, and Rotterdam. International Migration Review, 49(3), 633-658.

Delgado, M. (2018). Sanctuary cities, communities, and organizations: A nation at a crossroads. Reino Unido: Oxford University.

Doomernik, J. y Glorius, B. (2016). Refugee Migration and Local Demarcations: New Insight into European Localities. Journal of Refugee Studies, 29(4), 429-439. https://doi.org/10.1016/j.geoforum.2018.03.024

Dumitru, S. (2014). Qu'est-ce que le nationalisme méthodologique? Essai de typologie. Raisons politiques, 54(2), 9-22. https://doi.org/10.3917/rai.054.0009

Echazarro de Gregorio, A. (2009). Políticas públicas y segregación residencial de la población extranjera en la Comunidad de Madrid. Madrid: Fundación Alternativas.

Emilsson, H. (2015). A national turn of local integration policy: Multi-level governance dynamics in Denmark and Sweden. Comparative Migration Studies, 3(7), 3-16.

Fauser, M. (2008). Autoridades locales e integración política en ciudades de nueva inmigración: Los casos de Madrid y Barcelona. En R. Zapata-Barrero y G. Pinyol (Eds.), Los gestores del proceso de inmigración. Actores y redes de actores en España y Europa, (pp. 131-148). Colección Monografías. Barcelona: Fundación CIDOB.

Filomeno, F. A. (2016). Theories of Local Immigration Policy. Houndsmills: Palgrave Macmillan.

Filomeno, F. A. (2018). Global cities and multilevel immigration governance in latin America. En T. Caponio, P. Scholten y R. Zapata-Barrero (Eds.), The Roulledge handbook of The govennance of Migration and diversity in cities, (pp. 145-157). Londres/Nueva York: Routledge.

Furri, F. (2017). Villes-refuge, villes rebelles et néo-municipalisme. Plein droit, 115(4), 36. https://doi.org/10.3917/pld.115.0003

Garbaye, R. (2000). Ethnic minorities, cities, and institutions: A comparison of the modes of management of ethnic diversity of a French and a British city. En R. Koopmans y P. 
$20 \begin{aligned} & \text { Pensando la inmigración desde las instituciones de la ciudad } \\ & \text { Tshitshi Ndouba, K. }\end{aligned}$

Statham (Eds.), Challenging Immigration and Ethnic Relations Politics: Comparative European Perspectives, (pp. 283-311). Reino Unido: Oxford University Press.

Gilardoni, G., D’Odorico, M. y Carrillo, D. (2015). KING Knowledge for INtegration Governance. Evidence on migrants' integration in Europe. Milán: Fondazione ISMU.

Glazer, N. y Moynihan, D. P. (1963). Beyond the melting pot: The Negroes, Puerto Ricans, Jews, Italians, and Irish of New York City. Cambridge, MA: MIT Press.

Hatziprokopiou, P., Frangopoulos, Y. y Montagna, N. (2016). Migration and the city. Diversity, migrant economies and urban space Introduction. City, 20(1), 52-60. https://doi.org/10.1080/13604813.2015.1096054

International Organization for Migration. (2015). World Migration Report 2015. Recuperado de https://publications.iom.int/system/files/wmr2015_en.pdf

Izquierdo Escribano, A. y Noya, J. (1999). Lugares migratorios: Una propuesta teórica y metodológica para el análisis de la integración social de los inmigrantes. Migraciones, 6 , $19-42$.

Jørgensen, M. B. (2012). The Diverging Logics of Integration Policy Making at National and City Level. International Migration Review, 46(1), 244-278. https://doi.org/10.1111/j.1747-7379.2012.00886.x

Light, I. (1972). Ethnic Enterprise in America: Business and Welfare Among Chinese, Japanese, and Blacks. Berkeley/Los Ángeles/Londres: University of California Press.

Light, I. y Gold, S. (2000). Ethnic economies. San Diego, Ca: Academic Press.

Light, I., Sabagh, G., Bozorgmehr, M. y Der-Martirosian, C. (1994). Beyond the ethnic enclave economy. Social Problems, 41(1), 65-80.

Malheiros, J. (2002). Ethni-cities: Residential patterns in the northern european and Mediterranean metropolises-Implications for policy design. International Journal of population geography, 8(2), 107-134. https://doi.org/10.1002/ijpg.247

Moine, A. (2006). Le territoire comme un système complexe: Un concept opératoire pour l'aménagement et la géographie. L'Espace géographique, 2(35), 115-132. https://doi.org/10.3917/eg.352.0115

Moraes Mena, N. (2004). Entre el transnacionalismo y la relocalización: Un estudio del movimiento asociativo de los migrantes uruguayos en España. IV Congreso sobre la Inmigración en España. Girona, España.

Park, R.. E. y Burgess, E. (1921). Introduction to the science of sociology. Estados Unidos: University of Chicago Press.

Penninx, R. (2009). Decentralising integration policies: Managing migration in cities, regions and localities. Report. Policy Network. Recuperado de http://www.policynetwork.net/uploadedFiles/Publications/Publications/Decentralising\%20integration\%20 policies\%20FINAL\%20(Rinus\%20Penninx).pdf

Penninx, R. y Garcés-Mascareñas, B. (2016). Integration Policies of European Cities in Comparative Perspective: Structural Convergence and Substantial Differentiation. Migracijske i etničke teme, 32(2), 155-189. https://doi.org/10.11567/met.32.2.1 
Penninx, R. y Martiniello, M. (2006). Procesos de integración y políticas (locales): Estado de la cuestión y algunas enseñanzas. Revista Española de Investigaciones Sociológicas, (116), 123-156.

Pfirsch, T. y Semi, G. (2016). Segregation in the cities of the European Mediterranean. Méditerranée, (127), 15-23.

Portes, A. (2001). Inmigración y metrópolis: Reflexiones acerca de la historia urbana. Migraciones Internacionales, 1(1), 111-134.

Portes, A. y Jensen, L. (1989). The Enclave and the Entrants: Patterns of Ethnic Enterprise in Miami before and after Mariel. American Sociological Review, 54(6), 929-949. https://doi.org/10.2307/2095716

Sanyal, R. (2012). Refugees and the City: An Urban Discussion. Geography Compass, 6(11), 633-644. https://doi.org/10.1111/gec3.12010

Sassen, S. (1991). The Global City: New York, London, Tokyo. Princeton/Nueva Jersey: Princeton University Press.

Sassen, S. y Portes, A. (1993). Miami: A new global city? Contemporary Sociology, 22(4), 471-477.

Schiller, N. G. (2008). Beyond Methodological Ethnicity: Local and Transnational Pathways of Immigrant Incorporation. Malmö Institute for Studies of Migration and Ethnic Relations. Malmö: Malmö University.

Schiller, N. G., Basch, L. G. y Blanc, C. S. (1992). Towards a transnational perspective on migration: Race, class, ethnicity, and nationalism reconsidered. Estados Unidos: New York Academy of Sciences.

Schiller, N. G. y Çağlar, A. (2009). Towards a Comparative Theory of Locality in Migration Studies: Migrant Incorporation and City Scale. Journal of Ethnic and Migration Studies, 35(2), 177-202. https://doi.org/10.1080/13691830802586179

Schiller, N. G. y Çağlar, A. (2011). Locating Migration. Rescaling cities and migrants. Ithaca, NY: Cornell University Press.

Scholten, P. (2011). Constructing Dutch Immigrant Policy: Research-Policy Relations and Immigrant Integration Policy-Making in the Netherlands. The British Journal of Politics \& International Relations, 13(1), 75-92.

Scholten, P. (2013). Agenda dynamics and the multi-level governance of intractable policy controversies: The case of migrant integration policies in the Netherlands. Policy Sciences, 46(3), 217-236.

Scholten, P. (2016). Between National Models and Multi-Level Decoupling: The Pursuit of Multi-Level Governance in Dutch and UK Policies. Towards Migrant Incorporation. Journal of International Migration and Integration, 17(4), 973-994.

Scholten, P. y Penninx, R. (2016). The Multilevel Governance of Migration and Integration. En IMISCOE Research Series. Integration Processes and Policies in Europe. Contexts, Levels and Actors, (pp. 91-108). Dordrecht: Springer. 
$22 \begin{aligned} & \text { Pensando la inmigración desde las instituciones de la ciudad } \\ & \text { Tshitshi Ndouba, K. }\end{aligned}$

Scholten, P. (2019). Two worlds apart? Multilevel governance and the gap between national and local integration policies. En T. Caponio, P. Scholten y R. Zapata-Barrero, (Eds.), The Routledge Handbook to the Governance of Migration and Diversity in Cities, (pp. 157-167). Londres/Nueva York: Routledge.

Serra, A. (2007). La dimension local de la inmigración en Europa y en España. Datos básicos. En VV. AA. Inmigración y gobierno local: Experiencias y retos, (pp. 213-222). Barcelona: Fundación CIDOB.

Waldinger, R. (1985). The making of an immigrant niche. International Migration Review, (1), 1-30.

Zapata-Barrero, R. (2012). Coherencia entre gobernanza y política multinivel en materia de inmigración: España como laboratorio. Cuadernos Manuel Giménez, Extra (1), pp. 5363. 